\title{
In vitro phosphorylation as tool for modification of silk and keratin fibrous materials
}

\author{
Vadim Volkov ${ }^{1}$ - Artur Cavaco-Paulo ${ }^{1}$
}

Received: 11 February 2016 / Revised: 28 March 2016 / Accepted: 30 March 2016 / Published online: 13 April 2016

(C) Springer-Verlag Berlin Heidelberg 2016

\begin{abstract}
An overview is given of the recent work on in vitro enzymatic phosphorylation of silk fibroin and human hair keratin. Opposing to many chemical "conventional" approaches, enzymatic phosphorylation is in fact a mild reaction and the treatment falls within "green chemistry" approach. Silk and keratin are not phosphorylated in vivo, but in vitro. This enzyme-driven modification is a major technological breakthrough. Harsh chemical chemicals are avoided, and mild conditions make enzymatic phosphorylation a real "green chemistry" approach. The current communication presents a novel approach stating that enzyme phosphorylation may be used as a tool to modify the surface charge of biocompatible materials such as keratin and silk.
\end{abstract}

Keywords Silk fibroin · Hair keratin · Phosphorylation · Improved properties $\cdot$ Biomaterials

\section{Introduction}

Fibrous materials, whether natural or man-made, have been intensely studied and used in the variety of applications, such as civil engineering (Fangueiro et al. 2011), medicine (Fujihara et al. 2004), chemistry (Novoselova and Sirotkina 2006), and industry (Puglia et al. 2005), among others, due to their attractive and tunable properties. Nature demonstrates numerous examples where materials in a fibrous form are utilized to compose highly complex and multifunctional

Artur Cavaco-Paulo

artur@deb.uminho.pt

1 CEB - Centre of Biological Engineering, University of Minho, Campus of Gualtar, 4710-057 Braga, Portugal structures (reviewed in (Biagiotti et al. 2004; Lintz and Scheibel 2013). However, in some cases and under specific conditions, inherently non-fibrous proteins may form thin and extended fibrils, which are closely related to a number of pathological amyloid and prion states (Böckmann and Meier 2012).

Mulberry silk fibroin (SF) and, especially, hair keratin are probably one of the most recognized natural fibrous materials by a broad community. The silk of domesticated silkworm has been a basis of sericulture for many centuries (Khurana and Checker 2011), while hair keratin appears a target of an industry for cosmetic and care products (Weathersby and McMichael 2013). Since when the scientists and engineers began to realize the potential of SF and keratin in novel application fields, considerable amount of data regarding their structure, properties, and possible usages was reported (Zhou et al. 2001; Rockwood et al. 2011; McKittrick et al. 2012; Khosa and Ullah 2013; Kundu et al. 2013; Ghosh and Collie 2014; Lee et al. 2014; Yucel et al. 2014).

While working with some raw material, in most cases the researches wish to customize a final product, rendering (bio-)chemistry indispensable. In many cases, hazardous compounds such as hexafluoro-2-propanol (HFIP), hexafluoro-acetone (HFA), and 1-ethyl-3-(3dimethylaminopropyl)carbodiimide hydrochloride (EDC) are used at different steps of various SF-based products' customization. Sometimes ozone-mediated or $\gamma$-irradiation approaches are taken. (All of the above is reviewed in (Volkov et al. 2015a.) However, in the last decades numerous attempts were made to substitute the "conventional," purely chemical methods, by more environment-friendly, so-called green chemistry techniques (Höfer and Selig 2012). In that extent, enzymatically driven reactions attract significant attention, not only for being relatively non-hazardous or harsh treatments but also due to their high specificity towards the substrates (Demarche et al. 
2012). Working particularly on biomaterials like silk and keratin, it seems reasonable to employ enzymatic reactions for the diversification of the aforementioned substrates.

In vivo enzymatic phosphorylation is one of the beststudied post-translational modifications (PTMs) (Bononi et al. 2011), with fibrous material targets as no exception. In general, phosphorylation may induce conformational changes at both local and global levels via allosteric regulation of protein networks (Xin and Radivojac 2012). Other important aspects of this modification type, like its physiological stability (Westheimer 1987) and molecular interactions modulation (Mandell et al. 2007), have been presented previously. Specifically to SF (Zhang et al. 2006; Chen et al. 2010) and (solid) keratin fibers or filaments (Sun and Green 1978; Liao et al. 1995; Eckert and Yeagle 1996; Nakamura et al. 2002; Strnad et al. 2002; Pekny and Lane 2007), some pieces of evidence regarding in vivo phosphorylation were demonstrated in the past. However, only single works exist on enzymatic in vitro phosphorylation of both SF (Winkler et al. 2000) and keratin (Yeagle et al. 1990). In the first case, a transgenic short variant of spider silk was modified, whereas in the second bovine hoof-extracted keratin was processed. Both fibrous materials were phosphorylated in saluted forms; protein kinase A (PKA, EC 2.7.11.11) was the enzyme of choice. A general outlook on the proposed idea, including phosphorylation reaction, is shown in Fig. 1.

In the current communication, we summarize the experimental outcomes of three works, dealing with the reconstituted enzymatic reaction for modification of the chosen fibrous materials. It was of our interest to evaluate the possibility of enzyme-driven phosphorylation of the saluted wild-type SF and solid human hair keratin fiber. We clearly show that similarly to the natural processes, the phosphorylation of substrates, challenging to work with, can be performed in laboratory. This communication reports a more profound work on SF. Owing to this fact, we were able to elucidate the effects of phosphorylation on silk more extensively than on keratin.

\section{Phosphorylation feasibility and its implications on fibrous materials}

\section{- The feasibility of phosphorylation reaction}

\section{Silk fibroin}

Prior to attempting the phosphorylation reaction of SF, the very possibility of this modification was assessed in silico as described (Volkov et al. 2014). As such, 20 potential PKA recognition sites, of which 14 are found within hydrophobic region and are of primary importance, were detected (Fig. 2). SF was degummed and solubilized via the common protocols (Hu et al. 2010; Volkov et al. 2014), with the PKA-mediated phosphorylation followed. The optimized enzymatic reaction resulted in $\approx 60 \%$ of all predicted sites, as reported by colorimetric estimation of amounts of transferred phosphoryl group. The presence of the incorporated phosphate was further shown by FTIR (Fig. 3) for different blended fibroin films, elaborated by solvent casting and subsequent evaporation. $\mathrm{P}-$ $\mathrm{OH}$ deformation vibration-specific peak at $1039 \mathrm{~cm}^{-1}$ (Bellamy and Beecher 1952) supported the concept of in vitro phosphorylation.

\section{Hair keratin}

As opposed to silk, the keratin (in the solid form of hair tresses) was solely bleached and taken into kinase
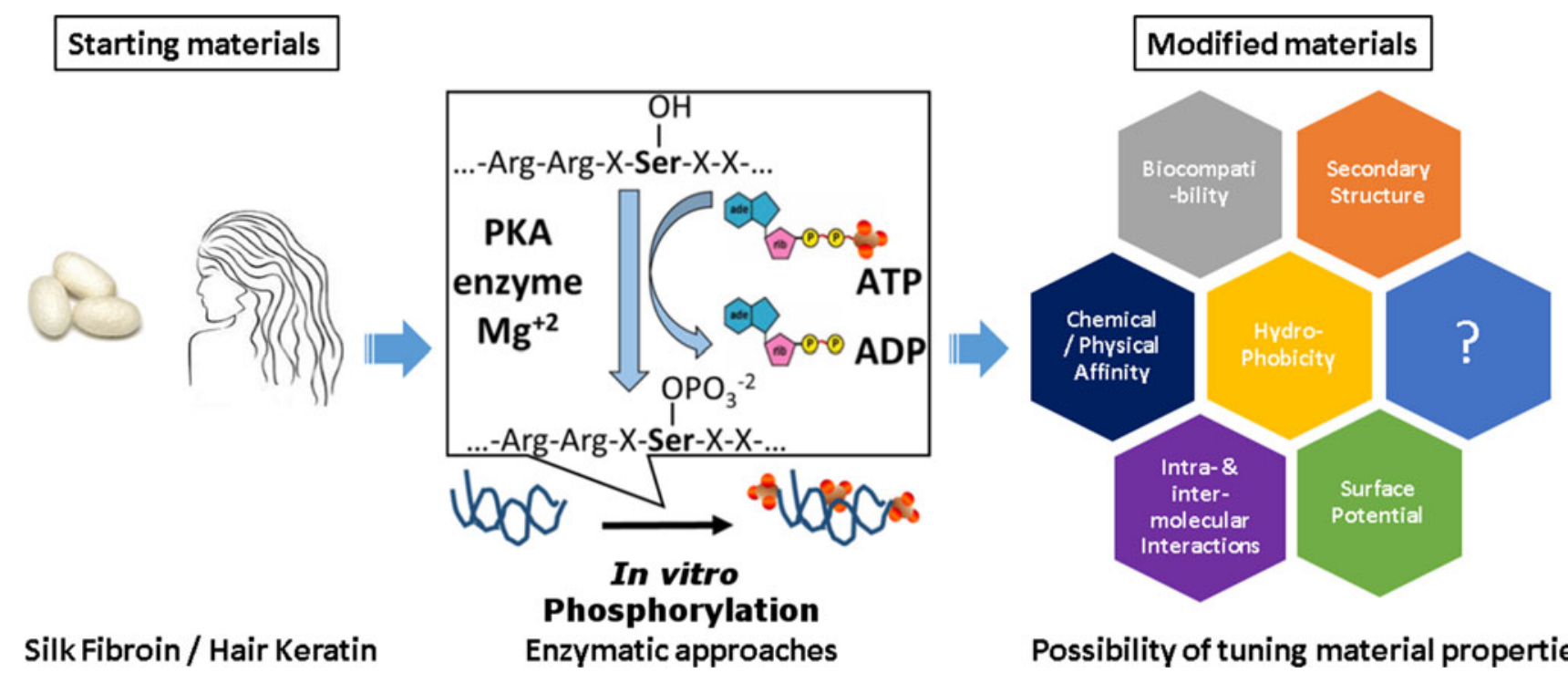

Fig. 1 In vitro enzymatic phosphorylation of fibrous materials opens a largely unexplored path of possibilities of modulating material properties 


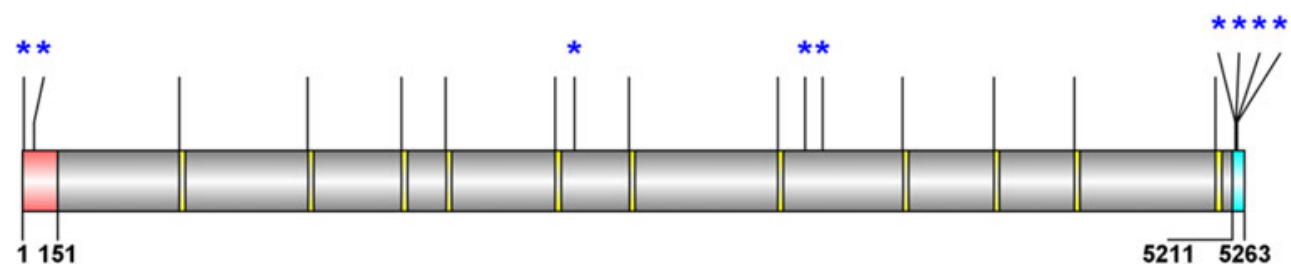

Fig. 2 The representation of predicted phosphorylated residues in B. mori SF. Asterisks denote the positions, estimated by the software, while the remaining sites were deduced upon protein sequence analysis using the consensus motif of PKA. Three major segment types, present in the protein, are indicated. Those include the $\mathrm{N}$-terminal acidic and $\mathrm{C}$ terminal basic domains, and 11 "amorphous" sequences. The rest

reaction (Volkov and Cavaco-Paulo 2016). Due to the specificity of its processing, no bioinformatics-based prediction regarding the number of possible phosphosites could be drawn. Yet, the previous successful attempt of keratin in vitro phosphorylation (Yeagle et al. 1990) made it possible to assume the feasibility of such modification. Indeed, diffuse reflection infrared spectroscopy (DRIFTS) and NMR assays of the modified keratin demonstrated although somewhat subtle, but present, phosphate (Fig. 4). The word "subtle" signifies the fact that, similarly to SF, where only 20 phosphorylationsuitable amino acids of $5263(\approx 0.38 \%)$ were found, keratin modification was accomplished to some low levels. For instance, instead of performing regular FTIR, a more sensitive DRIFTS was done (Armaroli et al. 2004). Accordingly, 20,700-20,900 transient scans were taken during NMR analysis to make the phospho-peak in Fig. 4b.

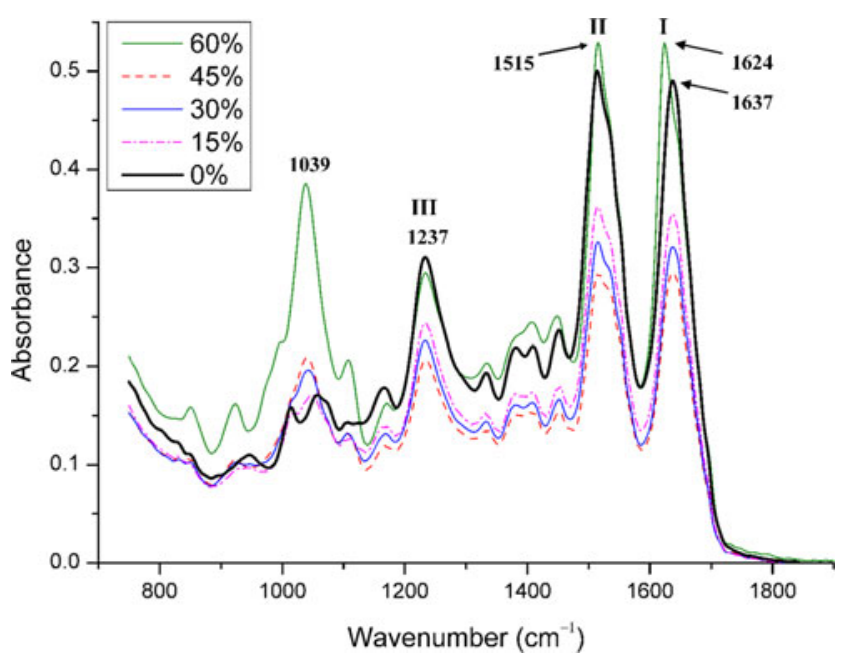

Fig. 3 FTIR spectra of SF films of various phosphorylation degrees. Main amide-related peaks $(I, I I$, and $I I I)$ and phosphorylation-derived peak are indicated. $0 \%, 15 \%, 30 \%, 45 \%$, and $60 \%$ indicate the final phosphorylation degrees. Reprinted with permission from Volkov et al. (2014). Copyright 2014 John Wiley \& Sons is repetitive crystalline region (Zhou et al. 2001). Borders of N- and Cterminal domains are indicated. This image was elaborated using the software, included with GPS v2.1 pack (Xue et al. 2008). Reprinted with permission from Volkov et al. (2014). Copyright 2014 John Wiley \& Sons

\section{- Phosphorylation implications on fibrous materials}

\section{Silk fibroin}

In general, $\beta$-sheet-forming proteins are difficult to maintain in aqueous solution due to the presence of hydrophobic domains that exclude water. Hydrophobic domains constitute a major part of silk heavy chain (H-chain) proteins and lead to the formation of strong intra- and inter-molecular $\beta$-related secondary structures, responsible for insolubility, high strength, and thermal stability of the silk fibers. Those secondary structures of silks, reflected in the polymorphic behavior of fibrous proteins, are a critical issue in the control of solubility of these proteins. Working on wild-type mulberry silk of domestic silkworm (H-chain protein), we corroborated previously published results of Winkler et al. (2000), while they had chosen a significantly different experimental system to work on. Winkler et al. demonstrated a system of transgenic spider silk fragment with the attached PKA recognition sequence. Their system was clearly tuned by (de-)phosphorylation in terms of $\beta$-sheet content and the resulting solubility. Notwithstanding, the transgenic system represented $\mathrm{a} \approx 25$ $\mathrm{kDa}$ protein, while our protein of target is estimated to be $\approx 350-391 \mathrm{kDa}$ in size. The latter undoubtedly means that potential impacts of phosphorylation (if any) are much harder to elucidate. Nevertheless, all the different works (preceding this communication and presented herein) show that innate hydrophobicity of silk as well as its self-assembly are highly dependent on polypeptide charges and steric hindrance factors, introduced by the phospho-group. This view, emerging from the currently presented works, is constituted by several experimental outcomes.

As such, FTIR-derived secondary structure analysis and DSC assessment of (phospho-)SF films depict the degree of $\beta$-sheet formation as a function of introduced phosphoryl group and the presence of phosphate-derived plasticization effect, respectively. Another structure-related finding was the enhanced ability of the modified films to swell. It was found that the material swelling is phosphorylation dependent and is highest when half of the starting SF is phosphorylated. 
a

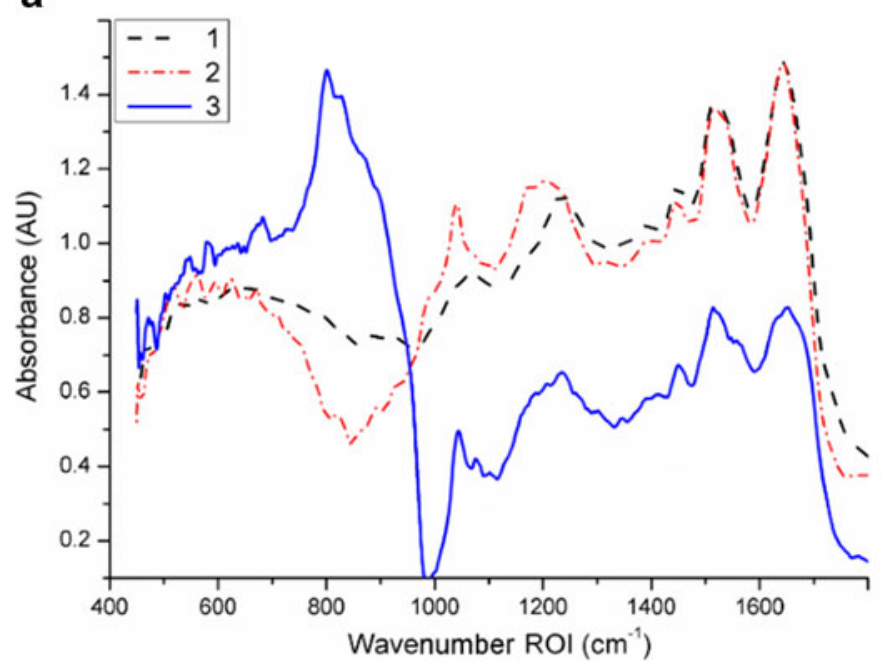

Fig. 4 Experimental evidence of hair keratin phosphorylation. a DRIFT spectra of hair dust, obtained from its outermost layers. 1 Virgin hair, 2 chemically damaged (bleached) hair, 3 bleached and subsequently phosphorylated hair. b NMR spectra of keratin peptides, liberated by

Besides that, a moderately increasing tendency to swell exists at a specific phosphorylation range; if the final material possesses higher phospho-content, swelling will decrease. The latter finding was explained from the basis of silk polymer relaxation degree and the ability to retain the solvent within the fibroin matrix.

When conducted at near neutral $\mathrm{pH}$, the release of incorporated model compounds, whether Piroxicam (Volkov et al. 2014) or Methotrexate (MTX) (Volkov et al. 2015b), underlined additional aspects of polymer-drug interactions. In the first case, phosphorylation decreased drug retention within the matrix, while no significant phosphorylationdependent trend was detected for the second case. Decreased hydrophobicity of the modified SF matrix promoted the release of mostly hydrophobic Piroxicam. As a compound of more prominent amphoteric character, MTX presents a highly $\mathrm{pH}$-dependent solubility and the ability to form interactions with the embedding material. Thus, the immediate impact of phosphate groups in the bulk phase may be masked or surpassed by the conditions of $\mathrm{pH}$. In addition, it was clarified that structural clustering of $\mathrm{SF}$ chains at acidic $\mathrm{pH}$ values generally leveled out the phosphorylation effects. Silk polypeptide chain collapse and its subsequent hydrophobic selfassociation at lower $\mathrm{pH}$ overcomes the phosphorylation repulsion effects due to its "modest" levels. Mechanistically, the acidified $\mathrm{pH}$-driven fibroin precipitation is very similar to the ongoing self-gelation of SF aqueous solutions with time (Matsumoto et al. 2006). The difference mainly stems from hydrophobic self-association and solvent exclusion speeds of the saluted material. Depending on SF concentration, solution ionic strength, and surrounding temperature, the precipitation process onset and/or propagation may range from hours to b
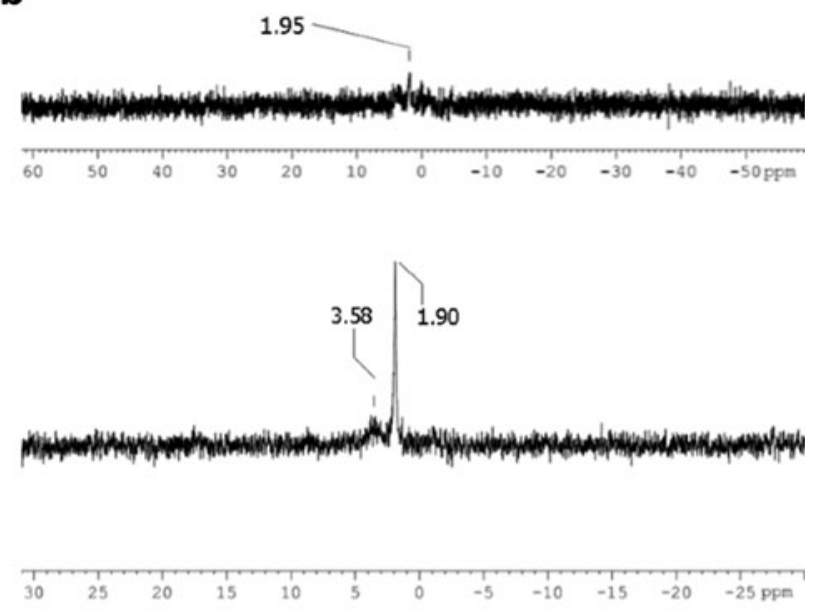

protease-mediated hair degradation. Top bleached hair only, bottom bleached and subsequently phosphorylated hair. Reprinted with permission from Volkov and Cavaco-Paulo (2016). Copyright 2016 Elsevier B. V

weeks. In our particular case, precipitates were started to be observed from the second week of storage of $\approx 2 \% \mathrm{w} / \mathrm{t}$ fibroin solution in $\mathrm{dH}_{2} \mathrm{O}$ at $4{ }^{\circ} \mathrm{C}$ and $\mathrm{pH} \approx 7.3$. $\mathrm{pH}$ drop to $\approx 3.5$ usually caused irreversible precipitation/gelation within $24 \mathrm{~h}$ at room temperature, regardless of the presence of the introduced phosphate. Although direct charge calculations for the modified silk demonstrated a tendency towards negative potential formation (and thus chain repulsion), as was noticed previously, this tendency is surpassed by acidification. Particularly important is the role of carboxyl groups (Glu and Asp) in determining fibroin chain interactions at acidic $\mathrm{pH}$ values. As a hydrophilic domain, the $\mathrm{N}$-terminus of the $\mathrm{H}$ chain of the fibroin is dominated by acidic amino acid side chains with the predicted $\mathrm{pI}$ of 4.59. Decreasing $\mathrm{pH}$ below 4.59 facilitates silk aggregation due to protonation of the carboxyl groups and thus promotes their intra- or inter-molecular interactions. Therefore, and similarly to the previous researches, we conclude that phosphorylation of SF caused a decrease in the hydrophobic content of the fold protein but did not completely inhibit $\beta$-sheet assembly.

Finally, in terms of cytotoxicity, it was shown that phospho-silk fibroin films seem not to promote cell attachment and, possibly, proliferation. Some previously cited works (Hezi-Yamit et al. 2009; Richards and Ivanisevic 2012) state that increased hydrophilicity disfavors cell attachment on the surfaces. Since the cellular membrane is known to be negatively charged from the outer side at physiological conditions (Emri et al. 1998; Klapperstuck et al. 2013), phospho-silk fibroin films will be prone to repel seeded cells. Thus, it can be solely stated with a high degree of confidence that lesser attachment will occur. Conclusions relating the surface charge and proliferation levels are thought to be too 
a

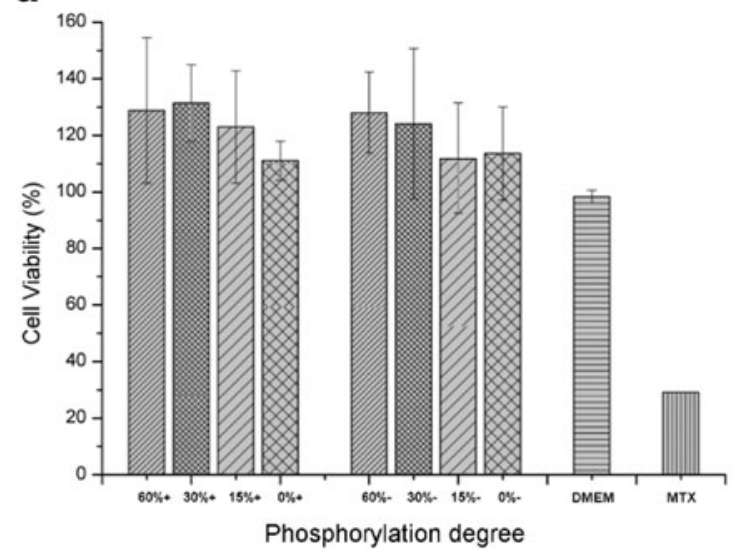

Fig. 5 The viability of the Caco-2 cell line, cultivated on lixiviates, derived from a 6-h incubation of growth medium with silk fibroin MTX-loaded films. a pH 7.2-cast films; b pH 3.5-cast films. "+" and "-" denote the MTX-loaded or MTX-devoid fibroin materials. DMEM = cell growth medium only, a positive control.

biased without any empirical confirmation. Furthermore, bioactivity evaluation of the MTX-loaded films on Caco-2 cells by indirect contact showed lower proliferation rate when the MTX release was higher (Fig. 5). Thus, films of higher phosphorylation were able to retain MTX for a longer time, and, importantly, denser films possessed lesser drug retention (Fig. 5b). This phenomenon was explained by some studies (Mallapragada et al. 1997; Kishida et al. 1998) and by us previously, as a drug exclusion during the formation of the hydrophobic $\beta$-sheet network.

\section{Hair keratin}

The third article reported that for hair keratin, the immediate outcome of phosphorylation is the enhanced affinity of a model cationic compound towards the phosphorylated material, as assessed by adsorption capacity $Q$ measurements of methylene blue (MB) dye b

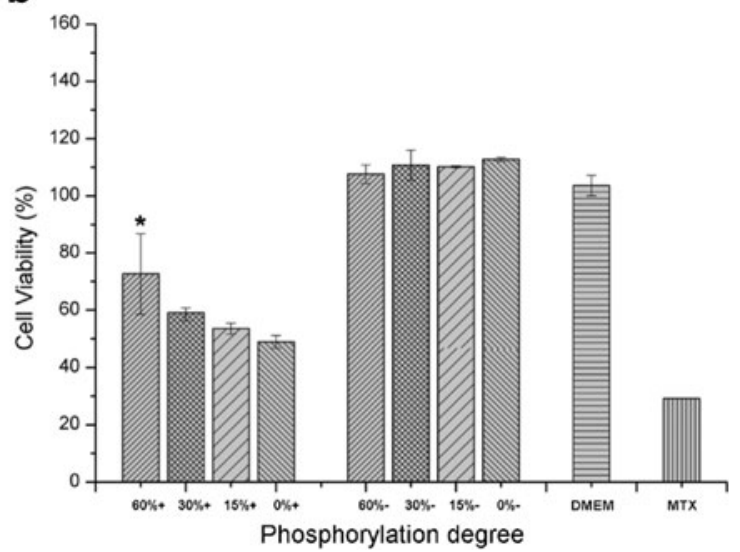

MTX = methotrexate at $0.2 \mathrm{mg} / \mathrm{ml}$ concentration, a negative control. Statistically significant difference is denoted by asterisk. Reprinted with permission from Volkov et al. (2015a). Copyright 2015 American Chemical Society

(Volkov and Cavaco-Paulo 2016). Since the enzymatic reaction was carried out on pre-formed solid material, only the considerations of adsorbent surface potential and adsorbate-adsorbent interactions were thoroughly studied and presented (Volkov and Cavaco-Paulo 2016).

Hair is known to possess high natural heterogeneity, for the most compositional, but also structural (Khan et al. 2014; Laatsch et al. 2014). During the experimental processing, human hair was extensively bleached and later phosphorylated. Structural and electric potentialrelated features, concurrently associated with the experimental treatments, are thought to include highly rough, perforated surface with areas of differential electrostatic potential energy. Since the later usage of a charged adsorbate, MB, as a model compound for clarification of aspects of the binding process, adsorbent surface was assumed to be energetically heterogeneous. This can be emphasized in Fig. 6, showing different models of

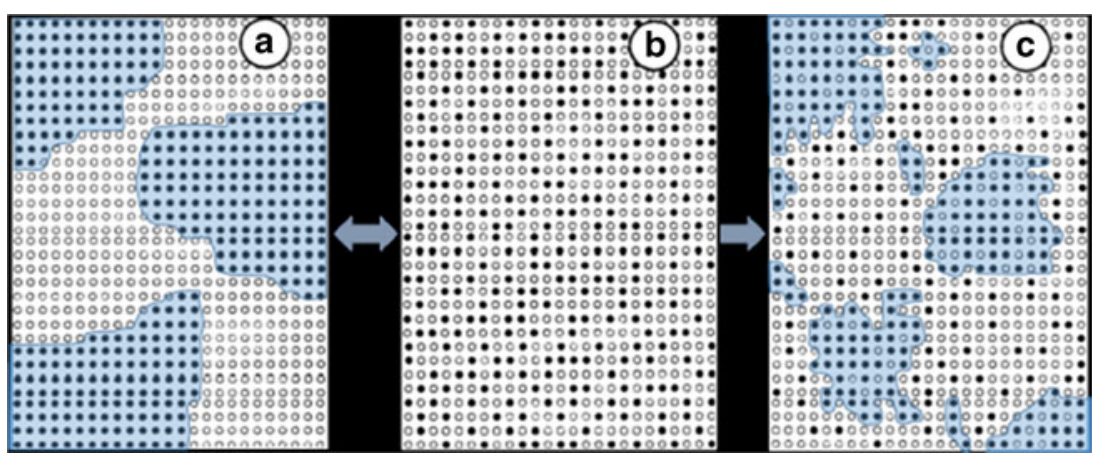

Fig. 6 Distribution models of the surface topography of adsorption centers for various energies on the solid surface. a "Patch-wise" type surface. b "Random" type surface. c "Intermediate" type surface. Two energetically distinct types of adsorption centers are marked with filled and blank circles. Where possible, the areas of similar energy potentials are marked by a cyan filler. The image is based on Charmas and Leboda (2000) 
Fig. 7 A view from the top of artificially colored 3D

visualization of the sites of highest dye affinity (dark-blue) and the bulk hair adsorbent volume (light-gray). a Nonmodified adsorbent. b Phosphomodified adsorbent. Scale bar, $7 \mu \mathrm{m}$. Reprinted with permission from Volkov and Cavaco-Paulo (2016). Copyright 2016 Elsevier B. V (color figure online)
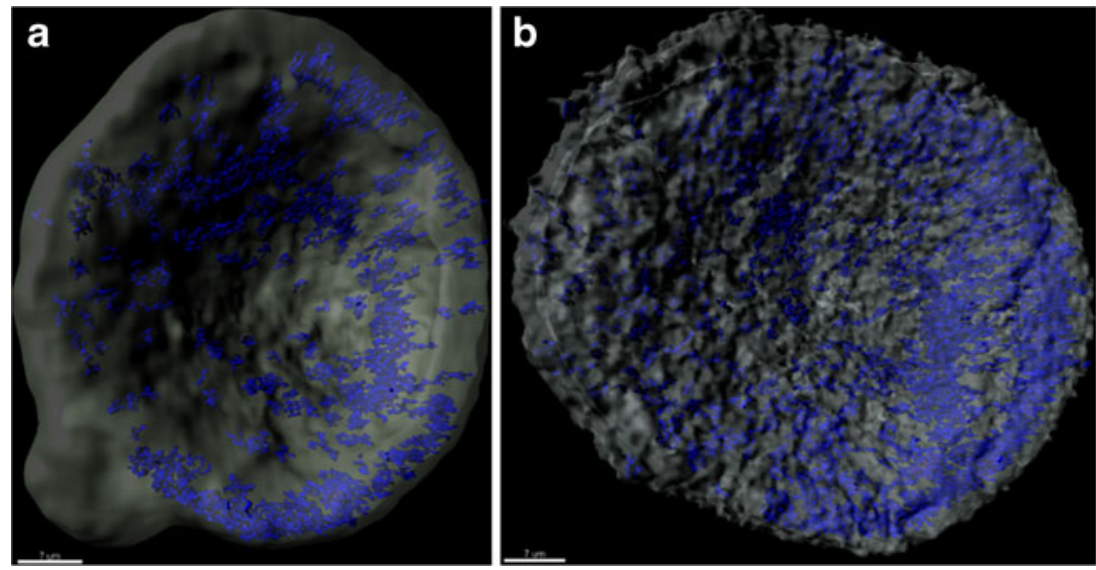

surface energetic heterogeneity (Charmas and Leboda 2000). We further assumed this surface to be of "intermediate" type. In a "patch-wise" type, the adsorption centers of the same properties are grouped in large patches. Those patches are considered to be large enough to constitute independent thermodynamic adsorption subsystems (Fig. 6a). On the contrary, the completely "random" distribution of adsorption centers causes the microscopic composition of the adsorbed phase in the surrounding of any center to be the same and identical to the average composition of the phase on the bulk solid surface (Fig. 6b). Thus, the patch-wise and random surface topographies represent two boundary cases for any solid adsorbent. In the particular case inspected here, along with many others, intermediate topography exists so that the correlation between the energies of the adsorption of the closed neighbors or even the neighbors of the closed adsorption sites may be evident (Fig. 6c). The latter model is strongly supported by experimental evidence, depicted in Fig. 7. The seemingly "pure" random distribution of dyebinding sites is disproved by the larger agglomerations of the bound $\mathrm{MB}$, pointing on the locations of elevated affinity towards MB. Practically, it means that while the distribution of these high-affinity locations may be random, the overall surface energetic heterogeneity of adsorbent falls within the pattern shown in Fig. 7c.

From calculations, based on $3 \mathrm{D}$ reconstruction of the bound MB (2D imprint is shown as Fig. 7), a $29.1 \%$ increase in the dye-binding sites as a result of phosphorylation was detected. The data acquired throughout the third study advocate for phosphorylation as a binder enhancer for the cationic MB. Various kinetic and thermodynamic modeling approaches envisage the beneficial role of phosphorylation in hair-dye interactions. The chosen results of kinetic pseudo-second order and Langmuir isothermal modelings are presented in Tables 1 and 2, respectively. Several important aspects of adsorption, such as the rate-limiting step of MB removal from a solution and its deposition on hair and thermodynamic modeling outcomes like the mean energy of adsorption, among others, were elucidated (Volkov and Cavaco-Paulo 2016). They made possible to conclude that the pseudo-second order (PSO) model, along with Langmuir isothermal model, characterizes best the hair-based adsorption system. Moreover, for the observed system, MB adsorption was an exothermic and energetically favored process, with entropy increase.

Summarizing, from the pairwise comparison of virtually all kinetic and thermodynamic parameters,
Table 1 Various estimated parameters of MB dye adsorption on different hair types

\begin{tabular}{lllll}
\hline Model applied & Hair types & Parameters, calculated from modeling & \\
\hline Kinetic pseudo-second order & & $Q_{\max }\left(\mathrm{mg} \mathrm{g}^{-1}\right)$ & $k_{2}\left(\mathrm{~g} \mathrm{mg}^{-1} \mathrm{~min}^{-1}\right)$ & $\overline{\mathrm{R}}^{2}$ \\
& $\mathrm{P}$ & 355.871 & $1.504^{*} 10^{-4}$ & 0.9924 \\
& $\mathrm{NP}$ & 273.224 & $1.067 * 10^{-4}$ & 0.9747 \\
Langmuir Isothermal & & $Q_{\max }\left(\mathrm{mg} \mathrm{g}^{-1}\right)$ & $K_{\mathrm{L}}\left(\mathrm{L} \mathrm{mg}^{-1}\right)$ & $\overline{\mathrm{R}}^{2}$ \\
& $\mathrm{P}$ & 374.531 & $46.675 * 10^{3}$ & 0.9647 \\
& $\mathrm{NP}$ & 322.580 & $49.301 * 10^{3}$ & 0.9868 \\
\hline
\end{tabular}

$\mathrm{NP}$ and $\mathrm{P}$ designate the non-modified or enzymatically modified hair

$Q_{\max }$ maximal dye absorption capacity, $k_{2}$ adsorption rate constant, $K_{\mathrm{L}}$ effective dissociation constant 
Table 2 Thermodynamic parameters of the MB dye adsorption onto different hair types

\begin{tabular}{|c|c|c|c|c|c|c|c|}
\hline \multirow[t]{2}{*}{ Hair type } & \multicolumn{3}{|c|}{ van’t Hoff equation-derived } & \multicolumn{4}{|c|}{$\Delta G$ at chosen temperature $\left(\mathrm{kJ} \mathrm{mol}^{-1}\right)$} \\
\hline & $\Delta H\left(\mathrm{~kJ} \mathrm{~mol}^{-1}\right)$ & $\Delta S\left(\mathrm{~J} \mathrm{~mol}^{-1}\right)$ & $R^{2}$ & $20^{\circ} \mathrm{C}$ & $30^{\circ} \mathrm{C}$ & $40^{\circ} \mathrm{C}$ & $50^{\circ} \mathrm{C}$ \\
\hline $\mathrm{P}$ & -29.106 & 19.991 & 0.9882 & -34.990 & -35.196 & -35.204 & -35.663 \\
\hline NP & -20.347 & 43.803 & 0.9573 & -33.306 & -33.463 & -33.967 & -34.620 \\
\hline
\end{tabular}

Calculated values of enthalpy $\Delta H$, entropy $\Delta S$, and Gibbs free energy $\Delta G$. NP and P designate the non-modified or enzymatically modified hair presented in Tables 1 and 2, it is seen that phosphorylation enhances the affinity of the hair to MB dye.

\section{Conclusions}

The three works, presented herein (Volkov et al. 2014, 2015b; Volkov and Cavaco-Paulo 2016), clearly demonstrate that natural fibrous materials of SF and keratin can undergo in vitro enzymatic phosphorylation. For hair keratin, the conclusion is even more striking, since it was processed as a solid fiber, and not a mixture of solubilized proteins, obtained by extraction. Both materials do undergo phosphorylation, yet to a very modest degree (Fig. 2 for SF and previous discussions on feasibility of enzymatic reaction for both SF and keratin). With this in mind, the impact on molecular weights of the modified macro-molecules is considered negligible. It is also assumed that in vitro phosphorylation per se does not induce or promote protein degradation. If one is expecting degradation, it is to occur during preparative steps for both materials (SF solubilization and keratin bleaching).

Yet, in vivo modification of the chosen fibrous materials still poses important questions. The biological meaning of post-translational modifications for both keratin (Sun and Green 1978; Liao et al. 1995; Eckert and Yeagle 1996; Nakamura et al. 2002; Strnad et al. 2002; Pekny and Lane 2007) and, particularly, SF (Zhang et al. 2006; Chen et al. 2010), still remain unclear. It is easily seen that the majority of available data correspond to keratin, and not silk. This fact stands to reason, as it has been established for a long time, that keratin filaments (along with vimentin, desmin, and neurofilament triplet proteins) play a pivotal role in eukaryotic cell biology, for instance in cytoskeleton remodeling and mechanical stability of cellular compartments like nucleus (Shih and Rothfield 2006). Nevertheless, the aspects of keratin filaments which function in cell biology are out of the scope of a current communication. Keratin filament self-organization and keratin-binding regulation are additional plausible explanations, analogous to those already postulated for different tissues (reviewed in Coulombe and Omary 2002). Previously cited literature only suggests the hypotheses of silk protein conformation remodeling and the alteration of binding to other factors. Unfortunately, the currently available data do not allow further conclusions for the role of silk in vivo phosphorylation. This lack of information is another important point that coerced us to conduct the work. It may be possible, to some extent, to extrapolate the obtained in vitro outcomes for in vivo systems, thus enriching our understanding of them.

Another key finding gleaned from the following studies is that phosphorylation made it possible to tailor various structural and physicochemical properties of the materials of interest. For both SF and keratin, the hallmark of obtained results unambiguously demonstrates that phosphorylation considerably alters the surfaces of polypeptide chains. For silk, alteration of secondary structure and density of forming hydrophobic packaging leads to varying retention of the incorporated drugs and decreased cell affinity. The latter may be important in material design, where cell attachment to implant surface is unwanted (Shen and Horbett 2001). For keratin, the improved affinity towards positively charged species, found in the surrounding solution, may implicate the feasibility of phosphorylation for hair pre-treatments in case of cosmetic applications, where cationic surfactants are commonly applied (Gavazzoni Dias et al. 2014) and references within).

\section{Further outlook on the feasibility of phosphorylation of fibrous materials}

Accordingly to the empiric data, presented herein, this enzyme-driven modification represents a valuable tool in the field of material design, especially where discriminative, sitespecific alterations are demanded. Another factor that strongly advocates for enzymatic processing is the mild nature of such treatment that is of importance in medical device fabrication and the related biocompatibility issues. Even a quantitatively minor modification, for instance, of solely $0.38 \%$ from the total amino acid amount, comprising SF H-chain, leads to considerable alteration of silk properties and the subsequent behavior of SF-based materials. Regarding hair keratin, while it was not possible to estimate the modification extent, keratin surface charge was shifted to lower values, influencing the formed electrostatic potential. Therefore, it is concluded that similarly to live systems, enzymatic phosphorylation may be successfully applied to tune various physicochemical properties in vitro. Indeed, convincing amount of various enzymatic 
processes have been implemented in a broad range of applications in recent decades (Jegannathan and Nielsen 2013). However, particularly for phosphorylation, its broad utilization in large-scale (industrial) applications is currently somewhat discouraged, due to significant cost of the enzyme and its decreased stability at the reaction conditions (if compared to purely chemical reagents). In cases where mass modification of specific functional groups or sites is needed, utilization of "canonical" chemistry may be still preferable. Future potential improvements (Kiss et al. 2013 and references within) in enzymatic stability and diversification of their consensus sequences, including de novo enzyme design strategies (Zanghellini 2014), may help overcome the obstacles, stated above.

\section{Compliance with ethical standards}

Funding This study was not funded by any external grant.

Conflict of interest The authors declare no conflict of interest.

Ethical approval This article does not contain any studies with human participants or animals performed by any of the authors.

\section{References}

Armaroli T, Bécue T, Gautier S (2004) Diffuse reflection infrared spectroscopy (DRIFTS): application to the in situ analysis of catalysts. Oil Gas Sci Technol 59:215-237

Bellamy LJ, Beecher L. 1952 The infra-red spectra of organo-phosphorus compounds. Part II. Esters, acids, and amines. J Chem Soc :17011706.

Biagiotti J, Puglia D, Kenny JM (2004) A review on natural FibreBased composites - part I. J Nat Fiber 1:37-68

Böckmann A, Meier BH (2012). Structural studies of protein fibrils by solid-state NMR. NMR of Biomolecules, Wiley-VCH Verlag GmbH \& Co. KGaA: 394-405.

Bononi A, Agnoletto C, De Marchi E, Marchi S, Patergnani S, Bonora M, Giorgi C, Missiroli S, Poletti F, Rimessi A, Pinton P (2011) Protein kinases and phosphatases in the control of cell fate. Enzym Res 2011:329098

Charmas B, Leboda R (2000) Effect of surface heterogeneity on adsorption on solid surfaces. Application of inverse gas chromatography in the studies of energetic heterogeneity of adsorbents. J Chromatogr A 886:133-152

Chen WQ, Priewalder H, John JP, Lubec G (2010) Silk cocoon of Bombyx mori: proteins and posttranslational modifications-heavy phosphorylation and evidence for lysine-mediated cross links. Proteomics 10:369-379

Coulombe PA, Omary MB (2002) 'Hard' and 'soft' principles defining the structure, function and regulation of keratin intermediate filaments. Curr Opin Cell Biol 14:110-122

Demarche P, Junghanns C, Nair RR, Agathos SN (2012) Harnessing the power of enzymes for environmental stewardship. Biotechnol Adv 30:933-953
Eckert BS, Yeagle PL (1996) Site-specificity of ethanol-induced dephosphorylation of rat hepatocyte keratins 8 and 18: a 31P NMR study. Cell Motil Cytoskeleton 33:30-37

Emri M, Balkay L, Krasznai Z, Trón L, Márián T (1998) Wide applicability of a flow cytometric assay to measure absolute membrane potentials on the millivolt scale. Eur Biophys J 28:78-83

Fangueiro R, Rosado K, Guise C, Rebelo R (2011). Fibrous based materials in sustainable civil and architectural applications - a review. International Conference On Engineering - Iceub2011., University of Beira Interior - Covilhã, Portugal, RepositoriUM, Uminho.

Fujihara K, Teo K, Gopal R, Loh PL, Ganesh VK, Ramakrishna S, Foong KWC, Chew CL (2004) Fibrous composite materials in dentistry and orthopaedics: review and applications. Compos Sci Technol 64:775-788

Gavazzoni Dias MF, de Almeida AM, Cecato PM, Adriano AR, Pichler J (2014) The shampoo $\mathrm{pH}$ can affect the hair: myth or reality? Int J Trichol 6:95-99

Ghosh A, Collie S (2014) Keratinous materials as novel absorbent systems for toxic pollutants. Def Sci J 64:209-221

Hezi-Yamit A, Sullivan C, Wong J, David L, Chen M, Cheng P, Shumaker D, Wilcox JN, Udipi K (2009) Impact of polymer hydrophilicity on biocompatibility: implication for DES polymer design. $\mathrm{J}$ Biomed Mater Res A 90:133-141

Höfer R, Selig M (2012). 10.02 - Green chemistry and green polymer chemistry. Polymer Science: A Comprehensive Reference. K. Matyjaszewski and M. Möller, Elsevier B.V. 10: 5-12.

Hu X, Wang X, Rnjak J, Weiss AS, Kaplan DL (2010) Biomaterials derived from silk-tropoelastin protein systems. Biomaterials 31 : $8121-8131$

Jegannathan KR, Nielsen PH (2013) Environmental assessment of enzyme use in industrial production - a literature review. J Clean Prod 42:228-240

Khan I, Maldonado E, Vasconcelos V, O'Brien SJ, Johnson WE, Antunes A (2014) Mammalian keratin associated proteins (KRTAPs) subgenomes: disentangling hair diversity and adaptation to terrestrial and aquatic environments. BMC Genomics 15:779

Khosa MA, Ullah A (2013) A sustainable role of keratin biopolymer in green chemistry: a review. J Food Process Beverages 1(1):4-8

Khurana P, Checker VG (2011) The advent of genomics in mulberry and perspectives for productivity enhancement. Plant Cell Rep 30:825838

Kishida A, Murakami K, Goto H, Akashi M, Kubota H, Endo T (1998) Polymer drugs and polymeric drugs $\mathrm{X}$ : slow release of $\mathrm{B}-$ fluorouracil from biodegradable $\operatorname{poly}(\gamma$-glutamic acid $)$ and its benzyl ester matrices. J Bioact Compat Polym 13:270-278

Kiss G, Çelebi-Ölçüm N, Moretti R, Baker D, Houk KN (2013) Computational enzyme design. Computational Enzyme Design. Angew Chem Int Ed 52:5700-5725

Klapperstuck T, Glanz D, Hanitsch S, Klapperstuck M, Markwardt F, Wohlrab J (2013) Calibration procedures for the quantitative determination of membrane potential in human cells using anionic dyes. Cytometry A 83:612-626

Kundu B, Rajkhowa R, Kundu SC, Wang X (2013) Silk fibroin biomaterials for tissue regenerations. Adv Drug Deliv Rev 65:457-470

Laatsch CN, Durbin-Johnson BP, Rocke DM, Mukwana S, Newland AB, Flagler MJ, Davis MG, Eigenheer RA, Phinney BS, Rice RH (2014) Human hair shaft proteomic profiling: individual differences, site specificity and cuticle analysis. Peer J 2:e506

Lee H, Noh K, Lee S, Kwon I-K, Han D-W, Lee I-S, Hwang Y-S (2014) Human hair keratin and its-based biomaterials for biomedical applications. Tissue Eng Regen Med 11:255-265

Liao J, Lowthert LA, Ku NO, Fernandez R, Omary MB (1995) Dynamics of human keratin 18 phosphorylation: polarized distribution of phosphorylated keratins in simple epithelial tissues. J Cell Biol 131: 1291-1301 
Lintz ES, Scheibel TR (2013) Dragline, egg stalk and byssus: a comparison of outstanding protein fibers and their potential for developing new materials. Adv Funct Mater 23:4467-4482

Mallapragada SK, Peppas NA, Colombo P (1997) Crystal dissolution-controlled release systems. II. Metronidazole release from semicrystalline poly(vinyl alcohol) systems. J Biomed Mater Res 36:125-130

Mandell DJ, Chorny I, Groban ES, Wong SE, Levine E, Rapp CS, Jacobson MP (2007) Strengths of hydrogen bonds involving phosphorylated amino acid side chains. J Am Chem Soc 129:820-827

Matsumoto A, Chen J, Collette AL, Kim UJ, Altman GH, Cebe P, Kaplan DL (2006) Mechanisms of silk fibroin sol-gel transitions. J Phys Chem B 110:21630-21638

McKittrick J, Chen P-Y, Bodde SG, Yang W, Novitskaya EE, Meyers MA (2012) The structure, functions, and mechanical properties of keratin. JOM 64:449-468

Nakamura A, Arimoto M, Takeuchi K, Fujii T (2002) A rapid extraction procedure of human hair proteins and identification of phosphorylated species. Biol Pharm Bull 25:569-572

Novoselova LY, Sirotkina EE (2006) Polyolefinic fibrous ion-exchange materials: properties and applications (review). Chem Sustain Dev 14:199-213

Pekny M, Lane EB (2007) Intermediate filaments and stress. Exp Cell Res 313:2244-2254

Puglia D, Biagiotti J, Kenny JM (2005) A review on natural fibre-based composites - part II. J Nat Fiber 1:23-65

Richards D, Ivanisevic A (2012) Inorganic material coatings and their effect on cytotoxicity. Chem Soc Rev 41:2052-2060

Rockwood DN, Preda RC, Yucel T, Wang X, Lovett ML, Kaplan DL (2011) Materials fabrication from Bombyx mori silk fibroin. Nat Protoc 6:1612-1631

Shen M, Horbett TA (2001) The effects of surface chemistry and adsorbed proteins on monocyte/macrophage adhesion to chemically modified polystyrene surfaces. J Biomed Mater Res 57:336-345

Shih YL, Rothfield L (2006) The bacterial cytoskeleton. Microbiol Mol Biol Rev 70:729-754

Strnad P, Windoffer R, Leube RE (2002) Induction of rapid and reversible cytokeratin filament network remodeling by inhibition of tyrosine phosphatases. J Cell Sci 115:4133-4148
Sun TT, Green H (1978) Keratin filaments of cultured human epidermal cells. Formation of intermolecular disulfide bonds during terminal differentiation. J Biol Chem 253:2053-2060

Volkov V, Cavaco-Paulo A (2016) Enzymatic phosphorylation of hair keratin enhances fast adsorption of cationic moieties. Int J Biol Macromol. doi:10.1016/j.ijbiomac.2015.12.082

Volkov V, Vasconcelos A, Sarria MP, Gomes AC, Cavaco-Paulo A (2014) Phosphorylation of silk fibroins improves the cytocompatibility of silk fibroin derived materials: a platform for the production of tuneable material. Biotechnol J 9:1267-1278

Volkov V, Ferreira AV, Cavaco-Paulo A (2015a) On the routines of wildtype silk fibroin processing toward silk-inspired materials: a review. Macromol Mater Eng 300:1199-1216

Volkov V, Sarria MP, Gomes AC, Cavaco-Paulo A (2015b) Phosphorylated silk fibroin matrix for methotrexate release. Mol Pharm 12:75-86

Weathersby C, McMichael A (2013) Brazilian keratin hair treatment: a review. J Cosmet Dermatol 12:144-148

Westheimer FH (1987) Why nature chose phosphates. Science 235:11731178

Winkler S, Wilson D, Kaplan DL (2000) Controlling beta-sheet assembly in genetically engineered silk by enzymatic phosphorylation/dephosphorylation, by. Biochemistry 39:14002

Xin F, Radivojac P (2012) Post-translational modifications induce significant yet not extreme changes to protein structure. Bioinformatics 28:2905-2913

Xue Y, Ren J, Gao X, Jin C, Wen L, Yao X (2008) GPS 2.0, a tool to predict kinase-specific phosphorylation sites in hierarchy. Mol Cell Proteomics 7:1598-1608

Yeagle PL, Frye J, Eckert BS (1990) Phosphorylation modulates keratin structure. Biochemistry 29:1508-1514

Yucel T, Lovett ML, Kaplan DL (2014) Silk-based biomaterials for sustained drug delivery. J Control Release 190:381-397

Zanghellini A (2014) De novo computational enzyme design. Curr Opin Biotechnol 29:132-138

Zhang P, Aso Y, Yamamoto K, Banno Y, Wang Y, Tsuchida K, Kawaguchi Y, Fujii H (2006) Proteome analysis of silk gland proteins from the silkworm, Bombyx mori. Proteomics 6:2586-2599

Zhou CZ, Confalonieri F, Jacquet M, Perasso R, Li ZG, Janin J (2001) Silk fibroin: structural implications of a remarkable amino acid sequence. Proteins 44:119-122 